\title{
Association between Depressive Symptoms and Metabolic Syndrome in Police Officers: Results from Two Cross-Sectional Studies
}

\author{
Tara A. Hartley, ${ }^{1,2}$ Sarah S. Knox, ${ }^{2}$ Desta Fekedulegn, ${ }^{1}$ Celestina Barbosa-Leiker, ${ }^{3}$ \\ John M. Violanti, ${ }^{4}$ Michael E. Andrew, ${ }^{1}$ and Cecil M. Burchfiel ${ }^{1}$ \\ ${ }^{1}$ Biostatistics and Epidemiology Branch, Health Effects Laboratory Division, National Institute for Occupational Safety and Health, \\ Morgantown, WV 26505, USA \\ ${ }^{2}$ Department of Community Medicine, School of Medicine, West Virginia University, Morgantown, WV 26506, USA \\ ${ }^{3}$ College of Nursing, Washington State University, Spokane, WA 99210, USA \\ ${ }^{4}$ Department of Social and Preventive Medicine, School of Public Health and Health Professions, State University of New York at Buffalo, \\ Buffalo, NY 14214, USA
}

Correspondence should be addressed to Tara A. Hartley, thartley@cdc.gov

Received 31 May 2011; Revised 22 November 2011; Accepted 29 November 2011

Academic Editor: Habibul Ahsan

Copyright (c) 2012 Tara A. Hartley et al. This is an open access article distributed under the Creative Commons Attribution License, which permits unrestricted use, distribution, and reproduction in any medium, provided the original work is properly cited.

\begin{abstract}
Policing is one of the most dangerous and stressful occupations and such stress can have deleterious effects on health. The purpose of this study was to examine the association between depressive symptoms and metabolic syndrome (MetSyn) in male and female police officers from two study populations, Buffalo, NY and Spokane, WA. Depressive symptoms were measured using the Center for Epidemiologic Studies-Depression (CES-D) scale. MetSyn was defined using the 2005 AHA/NHBLI guidelines. Analysis of covariance was used to describe differences in number of MetSyn components across depressive symptom categories. The number of MetSyn components increased significantly across categories of CES-D for Spokane men only ( $\mathrm{p}$-trend $=0.003$ ). For each 5unit increase in CES-D score, odds increased by $47.6 \%$ for having hypertriglyceridemia, by $51.8 \%$ for having hypertension, and by $56.7 \%$ for having glucose intolerance. Exploring this association is important since both are predictors of future chronic health problems and the results could be helpful in developing future gender-specific prevention and intervention efforts among police officers.
\end{abstract}

\section{Introduction}

Police officers are members of a unique occupational population whose exposure to potentially traumatic and life threatening events is part of their professional duty [1]. Additionally, officers routinely encounter organizational stressors, such as court appearances, excessive paperwork, and shift work [1]. Higher levels of perceived work stress have been associated with depression among older police officers [2]. Police officers have more adverse cardiovascular disease (CVD) risk factor levels and higher rates of CVD morbidity and mortality compared to other populations [3-6]. Work stress and depression are risk factors for the development of CVD and diabetes [2, 7-20].
The MetSyn is a clustering of abnormalities (abdominal obesity, hypertension, dyslipidemia, glucose intolerance) that has been associated with increased risk for subclinical atherosclerosis, CVD, diabetes, target organ damage, and total mortality [21]. The association between depression and the MetSyn has been studied over the past decade $[8,9,11$, $13,14,18,19,22-29]$, and more recently it has been suggested that MetSyn may be intermediate in the pathway between depression and CVD $[11,13,15,18,19,30$, 31]. Multiple pathophysiological mechanisms underlying the association between depression and MetSyn have been proposed. One mechanism is neuroendocrine and involves overstimulation of the hypothalamic-pituitary adrenal (HPA) axis which leads to excess cortisol secretion and has been 
associated with both the development of depression and the MetSyn components $[32,33]$. Another indirect pathway is through adverse health behaviors associated with depression, such as cigarette smoking, excessive use of alcohol, and a sedentary lifestyle, which may be used as coping responses and subsequently increase risk of MetSyn and CVD $[8,11,19]$.

The association between depression and MetSyn appears to be stronger in women, although the explanation for this gender difference is somewhat unclear $[11,15,18,23,25,26]$. One possible explanation involves the role of sex hormones. Overactivation of the HPA axis due to stress or depression may result in decreased levels of sex hormones like estrogen [34]. Decreased levels of estrogen, such as that occurs during menopause, can elevate visceral fat mass, a component of MetSyn [34].

Police officers are considered to be a relatively young and presumably healthy occupational group (i.e., healthy worker effect); yet they suffer from high levels of stress and CVD. The purpose of this study was to examine the association between depressive symptoms and the MetSyn separately among male and female police officers from two cross-sectional studies. No known studies were identified which examined this association in police officers. Participants are police officers from two geographically different locations, which may make the findings more generalizable to other groups of police officers. Based on the literature, the specific study hypotheses were the following: (1) depressive symptoms would be associated with a larger number of MetSyn components in male and female police officers, (2) the association of depressive symptoms with MetSyn and its components would differ between male and female police officers, and (3) the association between depressive symptoms and MetSyn would not differ by geographic location.

\section{Methods}

Participants included those from two studies at two separate locations: Buffalo, New York and Spokane, Washington.

2.1. Buffalo Cardio-Metabolic Occupational Police Stress (BCOPS) Study. The Buffalo Cardio-Metabolic Occupational Police Stress (BCOPS) Study, a cross-sectional study of urban police officers, was conducted between 2004 and 2009. The purpose of the BCOPS Study was to examine the association between police stressors and physiological and psychological health consequences. The Center for Health Research at the School of Public Health and Health Professions, State University of New York in Buffalo, NY served as the data collection site. All 710 active duty police officers from the Buffalo, NY, Police Department were invited to participate and the recruitment was ongoing for five years. No specific inclusion criteria were used for the study, other than that the participant be a sworn police officer and willing to participate in the study. Women officers who were pregnant at the time of examination were excluded $(n=2)$. All participants provided informed consent and all phases, testing, and reports of the study were approved by the State University of New York at Buffalo Internal Review Board and the National Institute for Occupational Safety and Health Human Subjects Review
Board. Of the 464 officers examined, 54 were removed from analyses (33 retired, 1 missing demographic information, 3 missing depressive symptoms, 17 missing MetSyn) leaving a final sample of 410 officers (304 men, 106 women; age range 21-66).

Questionnaires were administered to collect demographic and lifestyle information and psychosocial measures. Participants provided a medical history (including history of CVD) and a 12-hour fasting blood sample was collected by a certified phlebotomist. Medication use was ascertained through self-report and by inventory of current medications brought to the clinic. Blood parameters for the MetSyn were measured by standard laboratory techniques on the Beckman Coulter LX20 clinical chemistry analyzer and included a blood lipid panel for high-density lipoprotein cholesterol (HDL-C) and triglycerides and chemistry panels for glucose [35]. Anthropometric measures were conducted by trained clinic personnel. Waist circumference was measured as abdominal girth at the highest point of the iliac crest and the lowest point of the costal margin in the mid-axillary line. Blood pressure was determined using the average of the second and third of three separate measurements of resting systolic and diastolic blood pressure obtained with a standard sphygmomanometer.

2.2. Spokane Heart Study (SHS). The Spokane Heart Study (SHS) was a prospective, longitudinal study investigating the natural history of preclinical atherosclerosis. Details of the study have previously been reported [4]. Over a 12-year period, over 1,000 asymptomatic men and women were enrolled and followed every two years, generating an extensive data set of medical and lifestyle histories, blood and urine measurements, and Electron Beam Computed Tomography (EBCT) measures of coronary artery calcification. At baseline, all participants completed a comprehensive questionnaire which measured key behavioral, social, and lifestyle domains. Fasting blood samples and anthropometric measures were taken prior to the EBCT scan. Routine assays for blood chemistries, lipid profile, and hematology were performed [4]. The sample was almost equally divided between men and women. All participants provided informed consent and all phases, testing, and reports of the study were approved by the Washington State University Institutional Review Board. For the current study, data collection years 2003-2006, years most comparable to the BCOPS study, were used. Based on the questions, "what is the name of your current employer" and "what is your current job title," 134 police officers were identified. Of the 134 officers participating in the study, four were removed from analyses (two missing depressive symptoms and two missing MetSyn) leaving a final sample of 130 Spokane police officers (121 men, 9 women; age range 35-62).

2.3. Depressive Symptoms. In both studies, depressive symptoms were measured using the Center for Epidemiologic Studies-Depression (CES-D) scale. The CES-D is a short 20item scale designed to measure symptoms of depression (e.g., poor appetite, restless sleep, sadness) in the general population on a 4-point scale [36]. The 4-point scale represents 
how much each symptom occurred during the past seven days as follows: 0 (rarely or none of the time, less than 1 day), 1 (some or little of the time, 1-2 days), 2 (occasionally or a moderate amount of time, 3-4 days), and 3 (most or all of the time, 5-7 days). The CES-D is scored by reverse coding the appropriate items and summing the scores to obtain an overall score. Respondents with scores between 0 and 15 are unlikely to be clinically depressed, scores of 16 to 21 indicate mild to moderate depression, and scores of 22 or greater are associated with major depression [36]. The CESD has acceptable reliability, Cronbach alpha of 0.85 , and a split-half reliability ranging from 0.76 to 0.85 [34]. For the current study, the continuous CES-D and three categories of CES-D were used: low (0-8), middle (9-15), and high (16 or greater).

2.4. MetSyn. The MetSyn criteria were based on the National Cholesterol Education Program Adult Treatment Panel guidelines with recent modifications from the American Heart Association and the National Heart, Lung, and Blood Institute [37]. The individual MetSyn components included the following: (1) abdominal obesity (gender-specific waist circumference $\geq 102 \mathrm{~cm}$ in males, $\geq 88 \mathrm{~cm}$ in females), (2) hypertension (systolic blood pressure $\geq 130 \mathrm{~mm} \mathrm{Hg}$, diastolic blood pressure $\geq 85 \mathrm{~mm} \mathrm{Hg}$, or reported physiciandiagnosed hypertension and antihypertensive treatment), (3) reduced HDL-C (gender-specific fasting HDL-C $<40 \mathrm{mg} / \mathrm{dL}$ in men, $<50 \mathrm{mg} / \mathrm{dL}$ in women, or reported treatment with nicotinic acid or fibrates), (4) elevated triglycerides (fasting triglycerides $\geq 150 \mathrm{mg} / \mathrm{dL}$, or reported treatment with nicotinic acid or fibrates), and (5) glucose intolerance (fasting serum glucose $\geq 100 \mathrm{mg} / \mathrm{dL}$, or reported treatment for diabetes). Participants were categorized according to the number of MetSyn components (0-5). MetSyn was considered present in individuals with three or more components.

2.5. Statistical Methods. Descriptive statistics were used to characterize the study population. The number of MetSyn components and the prevalence of MetSyn and each individual component were calculated. Analyses of variance and covariance were used to estimate and describe the unadjusted and multivariate adjusted mean count of MetSyn components across CES-D categories. Tests for trend were obtained from linear regression analyses with continuous CES-D as the independent variables and count of MetSyn components as the dependent variable. Multivariate models were used to adjust for age, smoking status, and marital status. These covariates were chosen based on their association with depression and MetSyn and evidence in the literature. Logistic regression was used to calculate the odds for having each of the five MetSyn components by categories of CES-D score. Even though the tests of interaction for gender and location (Buffalo, Spokane) were not statistically significant, results were stratified in order to provide comparisons between locations and between male and female officers. All analyses were conducted using the SAS software, Version 9.2 (SAS Institute, Cary NC). Due to the small number of Spokane women in this study, the primary analysis of the association between CES-D and MetSyn could not be conducted in this group; however, descriptive statistics for these nine participants are reported.

\section{Results}

3.1. Demographic Characteristics. Demographic characteristics of the Buffalo, NY and Spokane, WA police officers are shown in Table 1. The Spokane officers were between six and eight years older than the Buffalo officers (Spokane range = $35-62$, Buffalo range $=21-66)$ and nearly all white $(1 / 130)$ compared to approximately $22 \%$ of Buffalo officers who were black or Hispanic. More Spokane officers had completed at least four years of college ( $44 \%$ versus 34\%) and the Spokane men held higher ranks (above police officer) than the Buffalo men or women $(67.7 \%$ versus $30.5 \%$ and $19.8 \%$, resp.). More male officers were married (78.2\% for Buffalo men, $91.7 \%$ for Spokane men) than female officers $(58.5 \%$ for Buffalo women, $55.6 \%$ for Spokane women). More female Buffalo officers were current smokers compared to the other three groups. The mean (SD) CES-D score was 7.5 (6.6) for Buffalo men, 6.6 (5.5) for Spokane men, 8.7 (8.2) for Buffalo women, and 5.8 (4.5) for Spokane women.

3.2. MetSyn and Its Components. The prevalence of each of the MetSyn components tended to be higher for Spokane officers compared to Buffalo officers (Table 2). The most notable differences were the higher prevalence for abdominal obesity, elevated triglycerides, and glucose intolerance in Spokane women compared to Buffalo women. The differences were not as large between Spokane and Buffalo men. The mean values for each of the five MetSyn components were higher for Spokane officers compared to Buffalo officers, most notably triglyceride levels for Spokane women $(165.3 \mathrm{mg} / \mathrm{dL})$ compared to Buffalo women $(88.4 \mathrm{mg} / \mathrm{dL})$. The prevalence of MetSyn ( $\geq 3$ components) was highest among Spokane officers, $37.2 \%$ for men and $33.3 \%$ for women, compared to Buffalo officers, $31.6 \%$ for men and $8.5 \%$ for women - half of the Buffalo women did not have any of the MetSyn components. Accordingly, the number of MetSyn components was higher among Spokane officers, 2.1 (1.4) for men and 1.7 (1.9) for women, compared to Buffalo officers, 1.9 (1.4) for men and 0.9 (1.1) for women.

\subsection{Association between Number of MetSyn Components and} Categories of CES-D Score. The unadjusted number of MetSyn components increased significantly across categories of CES-D for Spokane men (CES-D score 0-8 $=1.9$ (1.3), 9$15=2.6(1.3), \geq 16=3.1(1.6)$, p-trend $=0.003)$ (Table 3$)$. This association remained significant after adjustment for age ( $\mathrm{p}$-trend $=0.004)$ and multivariate adjustment for age, smoking, and marital status ( $p$-trend $=0.003$ ). No association was found between number of MetSyn components and CES-D score for Buffalo men or women. Similar analyses could not be conducted for Spokane women due to the low number of participants $(n=9)$. Excluding officers with reported CVD or previous treatment for CVD $(n=11)$ did not alter the associations appreciably (data not shown) 
TABLE 1: Demographic characteristics by location and gender.

\begin{tabular}{|c|c|c|c|c|c|c|c|c|}
\hline \multirow{3}{*}{ Characteristic } & \multicolumn{4}{|c|}{ Buffalo New York Police Officers $(N=410)$} & \multicolumn{4}{|c|}{ Spokane Heart Study Police Officers $(N=130)$} \\
\hline & \multicolumn{2}{|c|}{$\operatorname{Men}(N=304)$} & \multicolumn{2}{|c|}{ Women $(N=106)$} & \multicolumn{2}{|c|}{$\operatorname{Men}(N=121)$} & \multicolumn{2}{|c|}{ Women $(N=9)$} \\
\hline & $N$ & $\begin{array}{c}\text { Mean (SD) } \\
\text { or } \% \\
\end{array}$ & $N$ & $\begin{array}{c}\text { Mean }(\mathrm{SD}) \\
\text { or } \% \\
\end{array}$ & $N$ & $\begin{array}{c}\text { Mean }(\mathrm{SD}) \\
\text { or } \% \\
\end{array}$ & $N$ & $\begin{array}{c}\text { Mean }(\mathrm{SD}) \\
\text { or } \% \\
\end{array}$ \\
\hline Age & 304 & $40.8(7.7)$ & 106 & $40.8(5.9)$ & 121 & $49.1(6.1)$ & 9 & $47.3(2.7)$ \\
\hline$<40$ years & 131 & 43.1 & 43 & 40.6 & 10 & 8.3 & 0 & 0.0 \\
\hline $40-49$ years & 128 & 42.1 & 54 & 50.9 & 51 & 42.2 & 7 & 77.8 \\
\hline $50+$ years & 45 & 14.8 & 9 & 8.5 & 60 & 49.6 & 2 & 22.2 \\
\hline \multicolumn{9}{|l|}{ Ethnicity } \\
\hline White & 237 & 79.8 & 76 & 71.7 & 121 & 100.0 & 8 & 88.9 \\
\hline African American & 53 & 17.9 & 30 & 28.3 & 0 & 0.0 & 0 & 0.0 \\
\hline Hispanic & 7 & 2.4 & 0 & 0.0 & 0 & 0.0 & 1 & 11.1 \\
\hline \multicolumn{9}{|l|}{ Education } \\
\hline$<12$ years, high school/GED & 38 & 12.6 & 4 & 3.8 & 8 & 6.7 & 1 & 11.1 \\
\hline College $<4$ years & 160 & 53.0 & 65 & 61.3 & 59 & 49.2 & 4 & 44.4 \\
\hline College $4+$ years & 104 & 34.4 & 37 & 34.9 & 53 & 44.2 & 4 & 44.4 \\
\hline \multicolumn{9}{|l|}{ Marital status } \\
\hline Single & 29 & 9.6 & 23 & 21.7 & 0 & 0.0 & 3 & 33.3 \\
\hline Married & 236 & 78.2 & 62 & 58.5 & 110 & 91.7 & 5 & 55.6 \\
\hline Divorced & 37 & 12.3 & 21 & 19.8 & 10 & 8.3 & 1 & 11.1 \\
\hline \multicolumn{9}{|l|}{ Rank } \\
\hline Police officer & 207 & 69.5 & 84 & 79.3 & 39 & 32.2 & 6 & 66.7 \\
\hline Sergeant/lieutenant/captain & 51 & 17.1 & 11 & 10.4 & 47 & 38.8 & 1 & 11.1 \\
\hline Detective & 34 & 11.4 & 9 & 8.5 & 28 & 23.1 & 2 & 22.2 \\
\hline Executive & 5 & 1.7 & 1 & 0.9 & 7 & 5.8 & 0 & 0.0 \\
\hline Other & 1 & 0.3 & 0 & 0.0 & 0 & 0.0 & 0 & 0.0 \\
\hline \multicolumn{9}{|l|}{ Smoking status } \\
\hline Current & 40 & 13.2 & 28 & 27.2 & 4 & 3.3 & 1 & 11.1 \\
\hline Former & 64 & 21.2 & 31 & 30.1 & 40 & 33.1 & 2 & 22.2 \\
\hline Never & 199 & 65.7 & 44 & 42.7 & 77 & 63.6 & 6 & 66.7 \\
\hline Body mass index, $\mathrm{kg} / \mathrm{m}^{2}$ & 304 & $30.3(4.2)$ & 106 & $26.0(4.7)$ & 124 & $29.8(3.9)$ & 9 & $28.9(4.6)$ \\
\hline$<25$ & 23 & 7.6 & 53 & 50.0 & 5 & 4.1 & 0 & 0.0 \\
\hline $25-30$ & 137 & 45.1 & 36 & 34.0 & 65 & 53.7 & 7 & 77.8 \\
\hline$>30$ & 144 & 47.4 & 17 & 16.0 & 51 & 42.2 & 2 & 22.2 \\
\hline CES-D score & 304 & $7.5(6.6)$ & 106 & $8.7(8.2)$ & 122 & $6.6(5.5)$ & 9 & $5.8(4.5)$ \\
\hline Low score: $0-8$ & 203 & 66.8 & 68 & 64.2 & 88 & 72.7 & 7 & 77.8 \\
\hline Middle score: 9-15 & 70 & 23.0 & 21 & 19.8 & 22 & 18.2 & 2 & 22.2 \\
\hline High score (depression): $16+$ & 31 & 10.2 & 17 & 16.0 & 11 & 9.1 & 0 & 0.0 \\
\hline
\end{tabular}

nor did excluding officers taking antidepressant medications (available for Buffalo police officers only, $n=32$ ) (data not shown). Separate analyses excluding Buffalo female officers taking exogenous estrogen (birth control medication: $n=20$ or hormone replacement medication: $n=4$ ) also did not alter the association (data not shown).

3.4. Odds Ratios for Individual MetSyn Components by CES$D$ Score. Odds ratios were calculated for having each individual MetSyn component by CES-D score for Spokane men
(Table 4). For each 5-unit increase in CES-D score, the odds of having abdominal obesity increased by $42 \%$. Multivariate adjustment for age, smoking, and marital status slightly attenuated the association $(\mathrm{OR}=1.338,95 \% \mathrm{CI}=0.932-$ 1.917). For each 5-unit increase in CES-D score, the multivariate adjusted odds increased by $47.6 \%$ for having elevated triglycerides $(\mathrm{OR}=1.476,95 \% \mathrm{CI}=1.025-2.119)$, by $56.7 \%$ for having glucose intolerance $(\mathrm{OR}=1.567,95 \% \mathrm{CI}=1.056-$ 2.327 ), and by $51.8 \%$ for having hypertension $(\mathrm{OR}=1.518$, $95 \% \mathrm{CI}=1.035-2.221)$. 


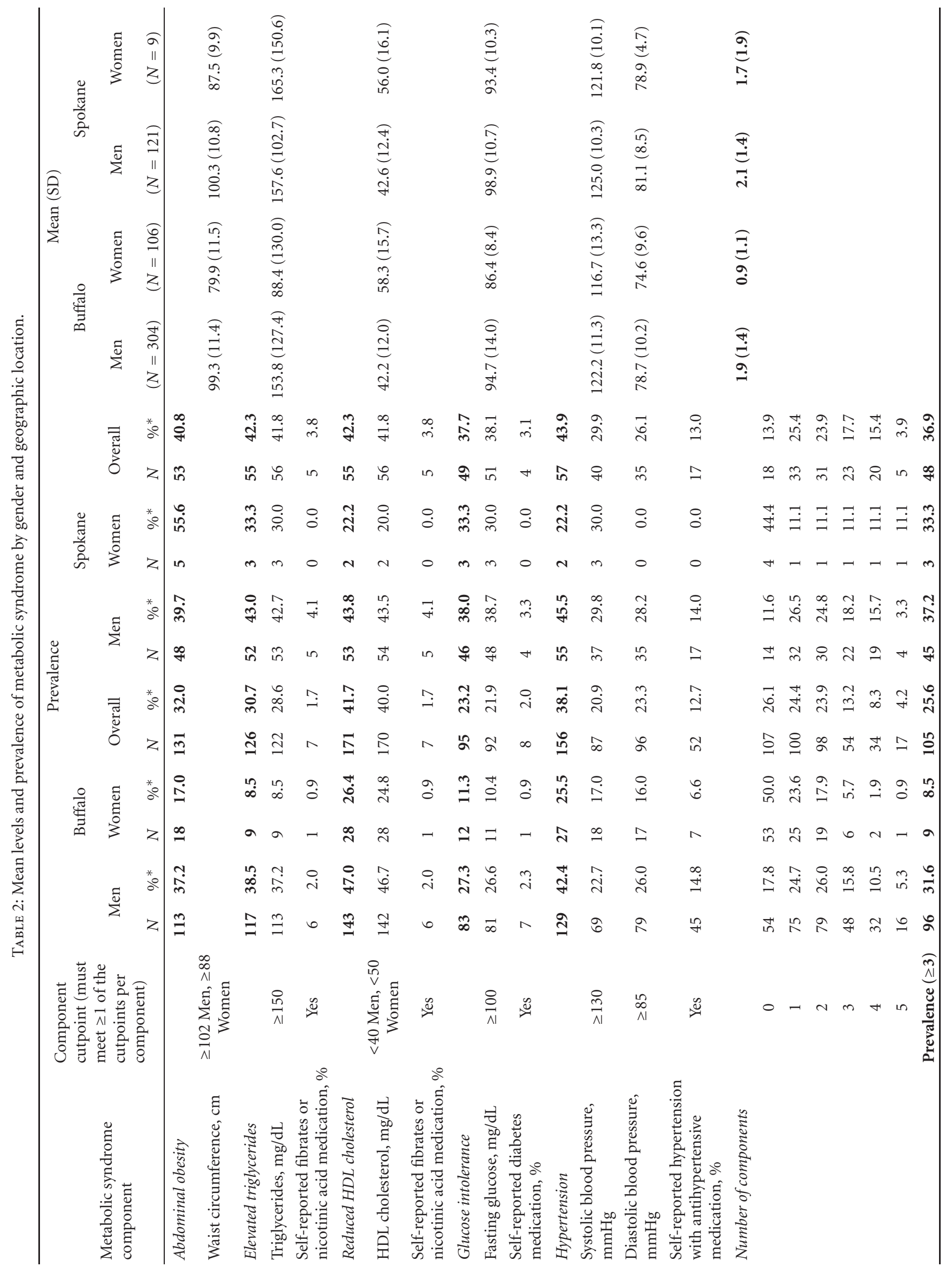


TABLE 3: Unadjusted and adjusted number of metabolic syndrome components by categories of CES-D score by location and gender.

\begin{tabular}{|c|c|c|c|c|c|c|c|c|c|}
\hline \multirow{3}{*}{ Categories of CES-D } & \multicolumn{9}{|c|}{ Number of metabolic syndrome components } \\
\hline & \multicolumn{3}{|c|}{ Unadjusted } & \multicolumn{3}{|c|}{ Age-adjusted } & \multicolumn{3}{|c|}{ Multivariate adjusted* } \\
\hline & $N$ & Mean (SD) & $P$ value** & $N$ & Mean (SD) & $P$ value** & $N$ & Mean (SD) & $P$ value** \\
\hline \multicolumn{10}{|l|}{ Buffalo men } \\
\hline $0-8$ & 203 & $1.8(1.4)$ & & 203 & $1.8(0.1)$ & & 202 & $1.9(0.1)$ & \\
\hline $9-15$ & 70 & $2.2(1.3)$ & 0.431 & 70 & $2.2(0.2)$ & 0.379 & 69 & $2.3(0.2)$ & 0.390 \\
\hline$\geq 16$ & 31 & $1.9(1.5)$ & & 31 & $2.0(0.2)$ & & 31 & $2.0(0.3)$ & \\
\hline \multicolumn{10}{|l|}{ Buffalo women } \\
\hline $0-8$ & 68 & $0.8(1.1)$ & & 68 & $0.8(0.1)$ & & 65 & $0.8(0.1)$ & \\
\hline $9-15$ & 21 & $1.1(1.4)$ & 0.376 & 21 & $1.1(0.2)$ & 0.377 & 21 & $1.2(0.3)$ & 0.610 \\
\hline$\geq 16$ & 17 & $0.9(0.9)$ & & 17 & $0.9(0.3)$ & & 17 & $0.7(0.3)$ & \\
\hline \multicolumn{10}{|l|}{ Spokane men } \\
\hline $0-8$ & 88 & $1.9(1.3)$ & & 88 & $1.9(0.1)$ & & 88 & $1.8(0.3)$ & \\
\hline $9-15$ & 22 & $2.6(1.3)$ & 0.003 & 22 & $2.6(0.3)$ & 0.004 & 21 & $2.7(0.4)$ & 0.003 \\
\hline$\geq 16$ & 11 & $3.1(1.6)$ & & 11 & $3.0(0.4)$ & & 11 & $3.0(0.5)$ & \\
\hline
\end{tabular}

${ }^{*}$ Adjusted for age, smoking status, and marital status.

${ }^{* *} P$ values are based on the continuous CES-D score.

TABLE 4: Unadjusted and adjusted odds ratios* for MetSyn components by CES-D score for Spokane men.

\begin{tabular}{lcccccc}
\hline \multirow{2}{*}{ MetSyn component } & \multicolumn{2}{c}{ Unadjusted $(N=121)$} & \multicolumn{2}{c}{ Age-adjusted $(N=121)$} & \multicolumn{2}{c}{ Multivariate adjusted ${ }^{* *}(N=120)$} \\
& Odds ratio & $95 \%$ CI & Odds ratio & $95 \%$ CI & Odds ratio & $95 \%$ CI \\
\hline Abdominal Obesity & 1.422 & $1.010-2.003$ & 1.396 & $0.985-1.977$ & 1.338 & $0.932-1.917$ \\
Elevated Triglycerides & 1.364 & $0.970-1.917$ & 1.449 & $1.020-2.064$ & 1.476 & $1.025-2.119$ \\
Reduced HDL-C & 0.975 & $0.699-1.357$ & 1.010 & $0.718-1.416$ & 1.030 & $0.730-1.456$ \\
Glucose Intolerance & 1.553 & $1.088-2.202$ & 1.456 & $1.005-2.119$ & 1.567 & $1.056-2.327$ \\
Hypertension & 1.396 & $0.985-1.968$ & 1.370 & $0.965-1.942$ & 1.518 & $1.035-2.221$ \\
\hline
\end{tabular}

* Odds ratios for having each metabolic syndrome component are based on a 5-unit change in CES-D score.

** Adjusted for age, smoking status, and marital status.

\section{Discussion}

One surprising finding of this study was the low CES-D scores in all groups (range 5.8-8.7) with between $64.2 \%$ and $77.8 \%$ of participants scoring in the low category $(0-8)$. This restricted range may have made the finding of a statistical association with MetSyn less likely. However, the prevalence of depression as indicated by a CES-D score $\geq 16$ was comparable with that reported in the Framingham Heart Study (10\% in men, $17 \%$ in women): $9.1 \%$ for Spokane men, $10.2 \%$ for Buffalo men, and $16 \%$ for Buffalo women, and the prevalence of MetSyn was notably higher for Spokane men and women and Buffalo men (range 31.6\%-37.2\%) compared to a recent study of US protective service workers $(26.1 \%)[38,39]$.

An association between depressive symptoms and number of MetSyn components was observed for male Spokane officers, partially supporting two of the study hypotheses: depression would be associated with a higher number of MetSyn components, and the association would be different between male and female police officers. The association between depressive symptoms and MetSyn found for the Spokane men extends to depressive symptoms in a nonclinical range (i.e., CES-D < 16) [11]. Previous studies have found similar findings in the association between depression and
MetSyn among a general population, among young and middle-aged women, among adult male twins, and among those at risk for CVD $[11,13,22,26,28,40,41]$. One study, noting the peculiarity of their finding, found an association between depressive symptoms and MetSyn in men but not in women [42]. Interestingly, the association found in the current study was significant only among the male officers at one location, Spokane, indicating that there may be potential factors not measured in this study that could have influenced the results, including traditional CVD risk factors such as physical activity and diet, and emerging CVD risk factors like inflammation and work stress [4, 20].

It is noteworthy that the Spokane officers were older and held higher ranks than the Buffalo officers. When the association between depressive symptoms and MetSyn was stratified on these two demographic variables, the association was strongest among the older ( $>49$ years, $n=59)$ versus younger Spokane men $(\leq 49, n=61)$, and lower-ranking (patrol officer, $n=39$ ) versus higher ranking Spokane men $(n=81)$ (data not shown). Yet, due to the small sample sizes for the stratified analyses the findings are reported with caution. Lower ranking police officers may experience high job demand and low job control. Perceived work stress has been associated with depression among older mostly male police officers, and high job strain has been significantly associated 
with depression among a national sample of working men [2, 43]. Vitaliano et al. suggested that chronic stress leads to depression which increases the risk of MetSyn and subsequently CVD [20].

For Spokane men, the odds of having four of the MetSyn components (abdominal obesity, hypertriglyceridemia, hypertension, glucose intolerance) increased by at least $40 \%$ for every 5-unit increase in CES-D score. The association with abdominal obesity was slightly attenuated by adjustment for age, smoking status, and marital status, while HDL$\mathrm{C}$ was not associated with depressive symptoms. This finding is supported by previous studies which have found depression to be associated with abdominal obesity, hypertriglyceridemia, hypertension, and glucose intolerance [11, 19, 29, $40,44,45]$. The association between depression and MetSyn and its components among these diverse populations suggests that the pathophysiology of both conditions may overlap; yet the biologic mechanisms may vary by MetSyn component. Several potential mechanisms could be considered [46].

Dysfunction of the hypothalamic-pituitary-adrenal (HPA) axis has been the most commonly cited mechanism for the association between depression and MetSyn [29, 46]. Fruehwald-Schultes et al. have shown that hyperinsulinemia activates the HPA axis and increases cortisol secretion [47] and this increase has been associated with depression, the MetSyn, and its individual components [11, 17, $32,33,46,48]$. Second, inflammation particularly elevated levels of C-reactive protein (CRP) have been associated with depressive symptoms in younger men, and in elderly men and women [49]. Inflammation has also been acknowledged as a key factor underlying development of MetSyn [22, 46]. Finally, as a means of coping depressed individuals may engage in poor health behaviors, such as a sedentary lifestyle, poor nutrition, cigarette smoking, excessive alcohol intake, or inadequate sleep, and these behaviors may lead to the development of MetSyn [11, 19]. Of these behaviors, only smoking status was available in the current study and it was the Buffalo women who had the highest percent of current and former smokers (57.3\%) compared to the other groups (all less than 37\%). However, consistent with previous studies, smoking status was included as a potential covariate in multivariate analyses although adjustment attenuated the association minimally across all groups.

Several limitations of the study should be noted. First, the CES-D provides a self-report measure of depressive symptoms as opposed to measuring clinical depression. Underreporting depressive symptoms is of concern with this occupational group as officers may not wish to disclose sensitive information. However, the CES-D has been widely used as a measure for assessing depressive symptoms in epidemiological studies. Second, this study was cross-sectional; therefore, we cannot determine whether depressive symptoms preceded the development of MetSyn and its components or make causal inferences. Others have reported that the MetSyn may lead to depression, specifically that hyperglycemia increases HPA axis activity causing arousal of the central nervous system thereby promoting development of depression [42]. Third, it is possible that participants in these two studies may not be representative of their respective police departments. However, for the Buffalo study comparisons between study participants and the Buffalo, NY Police Department resulted in similar frequency distributions for sex, age, and police rank. Finally, data are from two studies of police officers, thus potentially limiting the generalizability of the study findings to other police departments and other emergency responder populations.

One of the strengths of the current study was the ability to examine the association between depressive symptoms and MetSyn separately for men and women officers. A significant relationship has frequently been found among women with mixed results in men. Female police officers are an understudied occupational group and previous studies of police officers have typically included only male officers. Second, the study population was relatively young (mean age 49 for Spokane, 41 for Buffalo) and we were able to exclude those who have CVD or have received clinical interventions for CVD, enabling us to affirm that the relationship was unlikely attributable to CVD [11]. Third, we were able to exclude officers who were taking antidepressant medications and female officers who reported taking exogenous estrogen, although these exclusions did not alter the study findings. Finally, we used a highly standardized measure as our outcome, MetSyn [37]. In this study MetSyn was defined as the count of the number of components for each individual instead of limiting the assessment to presence/absence of MetSyn for the current study. This approach provides an interpretable measure of association and is more sensitive in the detection of associations [50].

In summary, depressive symptoms were associated with an increasing number of MetSyn components and with higher odds of having the individual MetSyn components among Spokane male police officers. This is the first study that we are aware of which has examined this relationship in police officers. Yet, it is particularly important to understand the association between depression and MetSyn among police officers given the high-stress nature of their job and the higher prevalence of CVD. Future studies should explore potential mediators for this association, including the role of the HPA axis and cortisol, inflammatory processes, and hyperinsulinemia, as these have been cited as potential mechanisms by others. It is also important to explore the genderspecific differences between male and female police officers. Depressive symptomatology and MetSyn are early indicators of future chronic health problems and the results could assist in the development of future gender-specific prevention and intervention efforts among police officers.

\section{Disclaimer}

The findings and conclusions in this report are those of the authors and do not necessarily represent the views of the National Institute for Occupational Safety and Health.

\section{Acknowledgment}

This work was supported by the National Institute for Occupational Safety and Health contract no. 200-2003-01580. 


\section{References}

[1] F. Deschamps, I. Paganon-Badinier, A. C. Marchand, and C. Merle, "Sources and assessment of occupational stress in the police," Journal of Occupational Health, vol. 45, no. 6, pp. 358364, 2003.

[2] R. R. M. Gershon, S. Lin, and X. Li, "Work stress in aging police officers," Journal of Occupational and Environmental Medicine, vol. 44, no. 2, pp. 160-167, 2002.

[3] W. D. Franke, S. L. Ramey, and M. C. Shelley, "Relationship between cardiovascular disease morbidity, risk factors, and stress in a law enforcement cohort," Journal of Occupational and Environmental Medicine, vol. 44, no. 12, pp. 1182-1189, 2002.

[4] B. R. Wright, C. Barbosa-Leiker, and T. Hoekstra, "Law enforcement officer versus non-law enforcement officer status as a longitudinal predictor of traditional and emerging cardiovascular risk factors," Journal of Occupational and Environmental Medicine, vol. 53, pp. 730-734, 2011.

[5] W. D. Franke, S. A. Collins, and P. N. Hinz, "Cardiovascular disease morbidity in an Iowa law enforcement cohort, compared with the general Iowa population," Journal of Occupational and Environmental Medicine, vol. 40, no. 5, pp. 441-444, 1998.

[6] J. M. Violanti, J. E. Vena, and S. Petralia, "Mortality of a police cohort: 1950-1990," American Journal of Industrial Medicine, vol. 33, pp. 366-373, 1998.

[7] T. Chandola, A. Heraclides, and M. Kumari, "Psychophysiological biomarkers of workplace stressors," Neuroscience and Biobehavioral Reviews, vol. 35, no. 1, pp. 51-57, 2010.

[8] B. E. Cohen, P. Panguluri, B. Na, and M. A. Whooley, "Psychological risk factors and the metabolic syndrome in patients with coronary heart disease: findings from the Heart and Soul Study," Psychiatry Research, vol. 175, no. 1-2, pp. 133-137, 2010.

[9] T. H. Heiskanen, L. K. Niskanen, J. J. Hintikka et al., "Metabolic syndrome and depression: a cross-sectional analysis," Journal of Clinical Psychiatry, vol. 67, no. 9, pp. 1422-1427, 2006.

[10] C. Victor Igna, J. Julkunen, H. Vanhanen, P. Keskivaara, and M. Verkasalo, "Depressive symptoms and serum lipid fractions in middle-aged men: physiologic and health behavior links," Psychosomatic Medicine, vol. 70, no. 9, pp. 960-966, 2008.

[11] L. S. Kinder, M. R. Carnethon, L. P. Palaniappan, A. C. King, and S. P. Fortmann, "Depression and the metabolic syndrome in young adults: Findings from the Third National Health and Nutrition Examination Survey," Psychosomatic Medicine, vol. 66, no. 3, pp. 316-322, 2004.

[12] H. S. Lett, J. A. Blumenthal, M. A. Babyak et al., "Depression as a risk factor for coronary artery disease: evidence, mechanisms, and treatment," Psychosomatic Medicine, vol. 66, no. 3, pp. 305-315, 2004.

[13] J. M. McCaffery, R. Niaura, J. F. Todaro, G. E. Swan, and D. Carmelli, "Depressive symptoms and metabolic risk in adult male twins enrolled in the National Heart, Lung, and Blood Institute Twin Study," Psychosomatic Medicine, vol. 65, no. 3, pp. 490-497, 2003.

[14] E. J. Mezick, M. Hall, and K. A. Matthews, "Are sleep and depression independent or overlapping risk factors for cardiometabolic disease?" Sleep Medicine Reviews, vol. 15, no. 1, pp. 51-63, 2011.

[15] C. Muhtz, B. C. Zyriax, T. Klähn, E. Windler, and C. Otte, "Depressive symptoms and metabolic risk: effects of cortisol and gender," Psychoneuroendocrinology, vol. 34, no. 7, pp. 1004-1011, 2009.

[16] A. Pan, M. Lucas, Q. Sun et al., "Increased mortality risk in women with depression and diabetes mellitus," Archives of General Psychiatry, vol. 68, no. 1, pp. 42-50, 2011.

[17] A. Rozanski, J. A. Blumenthal, and J. Kaplan, "Impact of psychological factors on the pathogenesis of cardiovascular disease and implications for therapy," Circulation, vol. 99, no. 16, pp. 2192-2217, 1999.

[18] S. Toker, A. Shirom, and S. Melamed, "Depression and the metabolic syndrome: gender-dependent associations," Depression and Anxiety, vol. 25, no. 8, pp. 661-669, 2008.

[19] V. Vaccarino, C. McClure, B. D. Johnson et al., "Depression, the metabolic syndrome and cardiovascular risk," Psychosomatic Medicine, vol. 70, no. 1, pp. 40-48, 2008.

[20] P. P. Vitaliano, J. M. Scanlan, J. Zhang, M. V. Savage, I. B. Hirsch, and I. C. Siegler, "A path model of chronic stress, the metabolic syndrome, and coronary heart disease," Psychosomatic Medicine, vol. 64, no. 3, pp. 418-435, 2002.

[21] G. M. Reaven, "Role of insulin resistance in human disease," Diabetes, vol. 37, no. 12, pp. 1595-1607, 1988.

[22] L. Capuron, S. Su, A. H. Miller et al., "Depressive symptoms and metabolic syndrome: is inflammation the underlying link?" Biological Psychiatry, vol. 64, no. 10, pp. 896-900, 2008.

[23] C. East, B. L. Willis, C. E. Barlow et al., "Depressive symptoms and metabolic syndrome in preventive healthcare: the cooper center longitudinal study," Metabolic Syndrome and Related Disorders, vol. 8, no. 5, pp. 451-457, 2010.

[24] A. Herva, P. Räsänen, J. Miettunen et al., "Co-occurrence of metabolic syndrome with depression and anxiety in young adults: The Northern Finland 1966 Birth Cohort Study," Psychosomatic Medicine, vol. 68, no. 2, pp. 213-216, 2006.

[25] A. Laudisio, E. Marzetti, F. Pagano, G. Pozzi, R. Bernabei, and G. Zuccalà, "Depressive symptoms and metabolic syndrome: selective association in older women," Journal of Geriatric Psychiatry and Neurology, vol. 22, no. 4, pp. 215-222, 2009.

[26] K. Räikkönen, K. A. Matthews, and L. H. Kuller, "The relationship between psychological risk attributes and the metabolic syndrome in healthy women: antecedent or consequence?" Metabolism: Clinical and Experimental, vol. 51, no. 12, pp. 1573-1577, 2002.

[27] K. Räikköonen, K. A. Matthews, and L. H. Kuller, "Depressive symptoms and stressful life events predict metabolic syndrome among middle-aged women: a comparison of World Health Organization, Adult Treatment Panel III, and International Diabetes Foundation definitions," Diabetes Care, vol. 30, no. 4, pp. 872-877, 2007.

[28] M. R. Skilton, P. Moulin, J. L. Terra, and F. Bonnet, "Associations between anxiety, depression, and the metabolic syndrome," Biological Psychiatry, vol. 62, no. 11, pp. 1251-1257, 2007.

[29] N. Vogelzangs, K. Suthers, L. Ferrucci et al., "Hypercortisolemic depression is associated with the metabolic syndrome in late-life," Psychoneuroendocrinology, vol. 32, no. 2, pp. 151159, 2007.

[30] A. Nicholson, H. Kuper, and H. Hemingway, "Depression as an aetiologic and prognostic factor in coronary heart disease: a meta-analysis of 6362 events among 146538 participants in 54 observational studies," European Heart Journal, vol. 27, no. 23, pp. 2763-2774, 2006.

[31] J. Zhang, R. Niaura, J. R. Dyer et al., "Hostility and urine norepinephrine interact to predict insulin resistance: the VA normative aging study," Psychosomatic Medicine, vol. 68, no. 5, pp. 718-726, 2006. 
[32] R. Rosmond and P. Björntorp, "The hypothalamic-pituitaryadrenal axis activity as a predictor of cardiovascular disease, type 2 diabetes and stroke," Journal of Internal Medicine, vol. 247, no. 2, pp. 188-197, 2000.

[33] F. Holsboer, "Stress, hypercortisolism and corticosteroid receptors in depression: implicatons for therapy," Journal of Affective Disorders, vol. 62, no. 1-2, pp. 77-91, 2001.

[34] P. Björntorp, "Do stress reactions cause abdominal obesity and comorbidities?” Obesity Reviews, vol. 2, no. 2, pp. 73-86, 2001.

[35] I. Mikolaenko, E. Benson, R. J. Konrad, C. Chaffin, C. A. Robinson, and R. W. Hardy, "Evaluation of the Beckman Coulter LX20 clinical chemistry analyzer," Laboratory Medicine, vol. 31, no. 7, pp. 387-393, 2000.

[36] L. S. Radloff, "The CES-D scale: a self-report depression scale for research in the general population," Applied Psychological Measurement, vol. 1, pp. 385-401, 1977.

[37] S. M. Grundy, J. I. Cleeman, S. R. Daniels et al., "Diagnosis and management of the metabolic syndrome: An American Heart Association/National Heart, Lung, and Blood Institute scientific statement," Circulation, vol. 112, no. 17, pp. 27352752, 2005.

[38] L. R. Wulsin, J. C. Evans, R. S. Vasan, J. M. Murabito, M. KellyHayes, and E. J. Benjamin, "Depressive symptoms, coronary heart disease, and overall mortality in the Framingham Heart Study," Psychosomatic Medicine, vol. 67, no. 5, pp. 697-702, 2005.

[39] E. P. Davila, H. Florez, L. E. Fleming et al., "Prevalence of the metabolic syndrome among U.S. workers," Diabetes Care, vol. 33, no. 11, pp. 2390-2395, 2010.

[40] J. Miettola, L. K. Niskanen, H. Viinamäki, and E. Kumpusalo, "Metabolic syndrome is associated with self-perceived depression," Scandinavian Journal of Primary Health Care, vol. 26, no. 4, pp. 203-210, 2008.

[41] E. M. Goldbacher, J. Bromberger, and K. A. Matthews, "Lifetime history of major depression predicts the development of the metabolic syndrome in middle-aged women," Psychosomatic Medicine, vol. 71, no. 3, pp. 266-272, 2009.

[42] H. Viinamaki, T. Heiskanan, S. M. Lehto et al., "Association of depressive symptoms and metabolic syndrome in men," Acta Psychiatry Scandinavica, vol. 120, pp. 23-29, 2009.

[43] E. R. Blackmore, S. A. Stansfeld, I. Weller, S. Munce, B. M. Zagorski, and D. E. Stewart, "Major depressive episodes and work stress: results from a national population survey," American Journal of Public Health, vol. 97, no. 11, pp. 2088 2093, 2007.

[44] K. Gil, P. Radziwiłłowicz, T. Zdrojewski et al., "Relationship between the prevalence of depressive symptoms and metabolic syndrome. Results of the SOPKARD Project," Kardiologia Polska, vol. 64, no. 5, pp. 464-469, 2006.

[45] N. Richter, G. Juckel, and H. J. Assion, "Metabolic syndrome: a follow-up study of acute depressive inpatients," European Archives of Psychiatry and Clinical Neuroscience, vol. 260, no. 1, pp. 41-49, 2010.

[46] R. S. McIntyre, N. L. Rasgon, D. E. Kemp et al., "Metabolic syndrome and major depressive disorder: co-occurrence and pathophysiologic overlap," Current Diabetes Reports, vol. 9, no. 1, pp. 51-59, 2009.

[47] B. Fruehwald-Schultes, W. Kern, W. Bong et al., "Supraphysiological hyperinsulinemia acutely increases hypothalamicpituitary-adrenal secretory activity in humans," Journal of Clinical Endocrinology and Metabolism, vol. 84, no. 9, pp. 3041-3046, 1999.
[48] M. Deuschle, B. Weber, M. Colla, M. Depner, and I. Heuser, "Effects of major depression, aging and gender upon calculated diurnal free plasma cortisol concentrations: a reevaluation study," Stress, vol. 2, no. 4, pp. 281-287, 1998.

[49] T. Liukkonen, S. Silvennoinen-Kassinen, J. Jokelainen et al., "The association between c-reactive protein levels and depression: results from the Northern Finland 1966 Birth Cohort Study," Biological Psychiatry, vol. 60, no. 8, pp. 825-830, 2006.

[50] D. Fekedulegn, M. Andrew, J. Violanti, T. Hartley, L. Charles, and C. Burchfiel, "Comparison of statistical approaches to evaluate factors associated with metabolic syndrome," Journal of Clinical Hypertension, vol. 12, no. 5, pp. 365-373, 2010. 


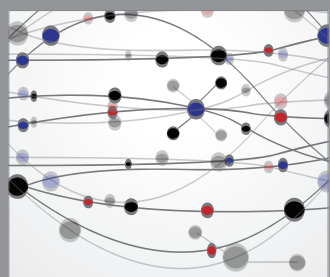

The Scientific World Journal


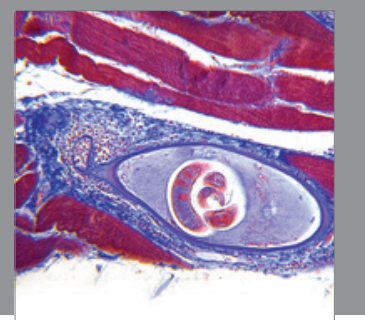

Gastroenterology

Research and Practice


\section{Hindawi}

Submit your manuscripts at

http://www.hindawi.com
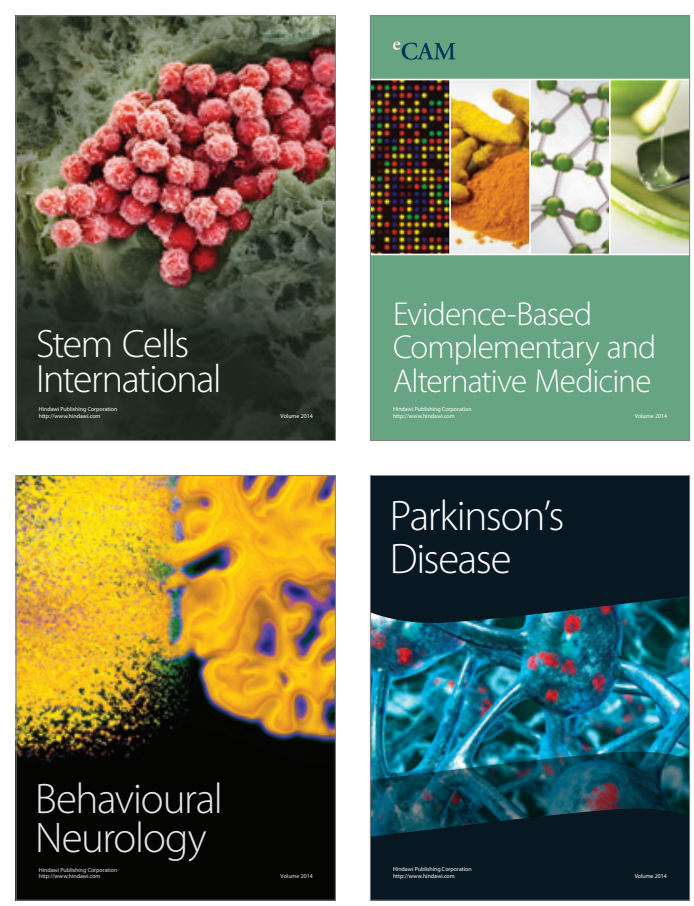

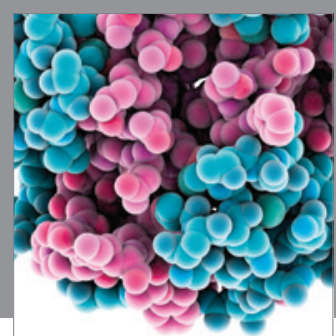

Journal of
Diabetes Research

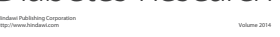

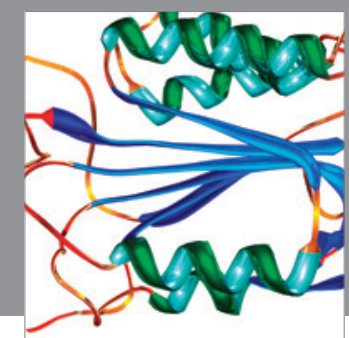

Disease Markers
